\title{
Microalbuminuria in relation to metabolic control and blood pressure in adolescents with type 1 diabetes
}

Maja Ješić, Silvija Sajić, Miloš Ješić, Mirjana Kostić, Amira Peco-Antić, Zoran Vujnović, Svetislav Necić

University Childrens Hospital, Belgrade, Serbia

Submitted: 27 June 2010

Accepted: 29 September 2010

Arch Med Sci 2011; 7, 6: 1037-1041

DOI: 10.5114/AOMS.2011.26617

Copyright ๑ 2011 Termedia \& Banach

\begin{abstract}
Introduction: The objective of this study was to assess the frequency of microalbuminuria and the relationship with other risk factors for the development of diabetic nephropathy.

Material and methods: Our cross-section study involved a group of 60 adolescence of both sexes, mean age $15.3 \pm 2.43$ years with mean duration of diabetes $7.74 \pm 3.44$ years. Albumin excretion rate was measured on $2-3$ samples of the first morning urine in the period below 6 months and persistent microalbuminuria was defined if its increased in two out of three urine specimens. Ambulatory blood pressure was monitored (ABPM, SpaceLabs 90207).

Results: Microalbuminuria developed in $13.3 \%$ of adolescents with mostly completed sexual development, statistically significantly poorer metabolic control (9.79\% vs. $8.7 \%)$ and higher BMI $\left(23.59 \mathrm{~kg} / \mathrm{m}^{2}\right.$ vs. $\left.20.85 \mathrm{~kg} / \mathrm{m}^{2}\right)$ than in the patients with normoalbuminuria. The mean night-time systolic blood pressure (SBP) was statistically significantly higher in microalbuminuric patients than in normoalbuminurics. The nocturnal dip was reduced in $41.7 \%$ of our patients; $38.5 \%$ of nondippers were in normoalbuminuric and $62.5 \%$ in microalbuminuric patients.

Conclusions: Diabetic adolescents require particular attention in order to minimize the factors such as high $\mathrm{HbA}_{1 c}$, elevated body mass index and night-time $\mathrm{SBP}$ in the development of incipient nephropathy.
\end{abstract}

Key words: microalbuminuria, ambulatory blood pressure monitoring, type 1 diabetes mellitus, adolescents.

\section{Introduction}

Diabetic nephropathy (DN) is one of the most serious complications of type 1 diabetes mellitus (DM1) and one of the most common causes of death in diabetics. The prevalence of DN among the diabetics who are affected by DM1 in childhood and the duration of disease lasting up to 30 years is about 15\% [1]. The prevalence of microalbuminuria (MA), the most serious predictive sign of nephropathy, is $2-20 \%$ during the first 10 years from the onset of diabetes, and about $30 \%$ at the end of the second decade of disease duration. Microalbuminuria is rarely present during the first $4-5$ years of diabetes. The presence of albumin in urine is caused by increased glomerular permeability and/or decreased reabsorption at the level of the proximal tubules. To confirm MA persistence, it is necessary to prove increased albumin excretion in two out of three urine specimens collected over a 3-6 month period [2, 3].

\author{
Corresponding author: \\ Miloš Ješić PhD \\ University Children's Hospital \\ Tiršova 1011000 Belgrade, \\ Serbia \\ Phone: +381 112060740 \\ Fax: +381 112684672 \\ E-mail: \\ milos.jesic@udk.bg.ac.rs
}


Although there is no unique explanation for the development of diabetic nephropathy and other microvascular complications, there are many risk factors that cause diabetic nephropathy or influence its progression: diabetes duration, metabolic control, hypertension, puberty, smoking, genetic factors, dyslipidaemia, etc. Increased blood pressure probably precedes microalbuminuria development and/or a kidney disease causing arterial hypertension. Progressive increase in blood pressure, although within the range of normal values, can be detected before the onset of persisting microalbuminuria, especially in adolescents with familial hypertension [4]. Ambulatory blood pressure monitoring (ABPM) is a sensitive, non-invasive method to diagnose the earliest changes of blood pressure in adolescents with DM1: absence of nocturnal dip, increased systolic and diastolic overload (defined by the frequency of blood pressure over the $95^{\text {th }}$ percentile matched for sex and age) and increase in mean arterial pressure [5-7]. Most likely both increased urine albumin excretion and blood pressure are manifestations of the same disease; also hypertension may be responsible for the acceleration of diabetic nephropathy progression to endstage renal failure [8].

The aim of our study was to assess the frequency of microalbuminuria in pubertal DM1 after a period of disease remission, and to determine the correlation between microalbuminuria and other risk factors for diabetic nephropathy, as well as their interrelationship: $\mathrm{HbA}_{1 \mathrm{c}}$, blood pressure, puberty, insulin doses, body mass index (BMI), and diabetes duration.

\section{Material and methods}

Using a cross-section study involving 60 adolescents (33 females, 27 males) of mean age 15.3 \pm 2.4 (11.0-19.7) years with DM1, with mean disease duration of $7.7 \pm 3.4$ (3.5-17.0), we evaluated the presence of microalbuminuria. The patients were treated at the University Children's Hospital of Belgrade, Serbia. All of the children were selected based on the following criteria: the duration of DM1 of over 3 years, patients aged over 11 years with preserved global renal function and who were not on ACE inhibitor renoprotective therapy. Mean annual $\mathrm{HbA}_{1 \mathrm{c}}$ values were determined based on three single findings each obtained after 2-4 months. $\mathrm{HbA}_{1 \mathrm{c}}$ was measured by turbidimetric immunoassay using Beckman Synchron CX5 Clinical System apparatus. In healthy persons, normal $\mathrm{HbA}_{1 \mathrm{c}}$ test results using this method range from $4.0 \%$ to $6.5 \%$. All the patients were tested for microalbuminuria in two out of three first morning urine samples collected within a 6-month period. Microalbuminuria was determined by the turbidimetric method using Architect Abbot appa- ratus where the defined normal value was below $15 \mathrm{mg} / \mathrm{l}$. The patients were defined as having persistent microalbuminuria if the obtained values were over $15 \mathrm{mg} / \mathrm{l}$ in two out of three samples. Using oscillometric type of SpaceLabs 90207 units and appropriate cuffs placed on the non-dominant arm, ABPM was performed after obtaining the second or third morning urine samples to assess microalbuminuria. Systolic blood pressure, diastolic blood pressure and mean arterial blood pressure were recorded and analysed by the ABPM program intended for calculating mean day-time, night-time and 24 -h values. The nocturnal dip expressed as a percentage of mean day-time blood pressure values was calculated using the formula: mean day-time BP-mean night-time BP $100 \% /$ mean day-time blood pressure. In our study, to assess the nocturnal dip, mean arterial pressure values were used. The nocturnal dip was defined as higher than 10\%.

For statistical data processing in our study we used the statistical package SPSS for Windows. The obtained data were presented as mean \pm SD. The comparison between the two independent groups was evaluated using the independent two-sample Student's t-test or Mann-Whitney U-test when appropriate. The default of $\alpha<0.05$ specified the level of significance in all of the tests. The correlation between the numerical variables was calculated by Spearman's correlation coefficient $(\rho)$. Univariate analysis for predictors of microalbuminuria was performed using simple logistic regression.

\section{Results}

Demographic characteristics of the patients are presented in Table I. Eight patients (13.3\%) met the criteria for microalbuminuria. All of the adolescents with microalbuminuria were either in stage II-IV of puberty (25\%) or had completed pubertal development (75\%).

A significantly higher mean value of nocturnal systolic blood pressure was observed in patients with microalbuminuria in comparison to normoalbuminuric patients $(t=2.161, p<0.05)$. With respect to all other BP values there was no statistically significant difference $(p>0.05)$ (Table II).

We found a statistically significant correlation between the night-time systolic BP and microalbuminuria, indicating the association between increased night-time BP and urine albumin excretion and vice versa $(\rho=0.379, p=0.00)$.

The duration of diabetes and insulin doses were in a statistically significant correlation with systolic night-time BP ( $\rho=0.313, p=0.01 ; \rho=0.269$, $p=0.03)$ and diastolic night-time BP $(\rho=0.301$, $p=0.02 ; \rho=0.331, p=0.01$ ). No correlation was found between urine albumin excretion, the duration of diabetes, insulin doses and day-time BP. 
The nocturnal dip was reduced in $41.7 \%$ of patients; $38.5 \%$ of normoalbuminuric and $62.5 \%$ of microalbuminuric patients were non-dippers. A statistically significant difference was found in the mean values of patients' ages ( $t=2.124, p=0.04)$ and $\mathrm{BMI}(t=2.643, p=0.01)$ between normoalbuminuric and microalbuminuric patients without a nocturnal dip of mean arterial pressure. There was no statistically significant difference $(p>0.05)$ regarding other parameters $\left(\mathrm{HbA}_{1 \mathrm{c}}\right.$, duration of diabetes and insulin doses).

Using univariate logistic regression, in our patients statistically significant risk factors for the development of microalbuminuria were high $\mathrm{HbA}_{1 c}$ (hazard ratio $(H R)=1.52$, 95\% confidence interval (CI): 0.97-2.40), BMI ( $\mathrm{HR}=1.26,95 \% \mathrm{Cl}: 1.00-1.60)$ and SBP (HR $=1.10,95 \% \mathrm{Cl}: 1.00-1.20)$ (Table III).

\section{Discussion}

Recent extensive studies have shown a decline in incidences of diabetic nephropathy $[9,10]$. In a study of diabetic patients aged below 18 years of age, with diabetes duration lasting over 5 years, the European Epidemiology Study of Diabetic Complications (EDC Study) reported a prevalence of diabetics with microalbuminuria of $14 \%$, whereas in other studies the reported prevalence of diabetics with microalbuminuria is $2-20 \%$ [11]. In our study, microalbuminuria developed in eight patients (13.3\%).

In DM1 prepubertal children the problem of microvascular complications is rarely seen, in comparison to its rising prevalence during and after puberty [12]. The Oxford Regional Study showed that at initial stages of diabetes the incidence of microalbuminuria was higher in pubertal than in prepubertal patients [13]. These results support the view that endocrine or metabolic factors (growth hormone or IGF-1) can trigger or accelerate renal damage [13]. Our microalbuminuric patients had mean diabetes duration longer than normoalbuminuric patients (9.37 vs. 7.49 years) and $75 \%$ of microalbuminuric patients were in the late pubertal stage. However, once puberty is achieved, the prepubertal years of diabetes contribute as an additional risk for microalbuminuria, but longer followup is needed to confirm these observations in our study.

We assessed mean $\mathrm{HbA}_{1 \mathrm{c}}$ (\%) annual value as the parameter reflecting the metabolic control of the disease. In our patients with microalbuminuria mean $\mathrm{HbA}_{1 c}$ was higher $(9.79 \%)$ than in the patients with normoalbuminuria (8.7\%); there was a statistically significant difference between these two groups $(p<0.05)$. Other studies have also shown that poor metabolic control is a risk factor in the development of microalbuminuria directly associated with hyperfiltration and renal hyper-
Table I. Clinical characteristics of normoalbuminuric and microalbuminuric DM1 patients

\begin{tabular}{|lcc|}
\hline Parameter & $\begin{array}{c}\text { NA patients } \\
N=52\end{array}$ & $\begin{array}{c}\text { MA patients } \\
N=8\end{array}$ \\
\hline Age [years] & $15.14 \pm 2.49$ & $16.45 \pm 1.67$ \\
\hline $\begin{array}{l}\text { Pubertal stages } \\
\text { I/II-IV/V [\%] }\end{array}$ & $3.8 / 48.1 / 48.1$ & $0 / 25 / 75$ \\
\hline Insulin dose [U/kg] & $1.15 \pm 0.28$ & $1.17 \pm 0.05$ \\
\hline HbA $_{1 \mathrm{c}}[\%]^{*}$ & $8.70 \pm 1.41$ & $9.79 \pm 1.64$ \\
\hline Duration of DM [years] $^{*} 7.49 \pm 3.50$ & $9.37 \pm 2.57$ \\
\hline${\text { BMI* }\left[\mathrm{kg} / \mathrm{m}^{2}\right]}^{2}$ & $20.85 \pm 3.41$ & $23.59 \pm 3.35$ \\
\hline AEU $^{*}[\mathrm{mg} / \mathrm{l}]$ & $6.53 \pm 3.60$ & $22.72 \pm 9.34$ \\
\hline
\end{tabular}

$D M$ - diabetes mellitus, $B M I$ - body mass index, $A E U$ - albumin excretion rate, ${ }^{*} p<0.05$ between normoalbuminuric (NA) and microalbuminuric (MA) patients

Table II. Descriptive statistical indices for day-time, night-time and 24-h values of ABPM in patients with and without microalbuminuria

\begin{tabular}{|lccc|}
\hline BP $[\mathrm{mmHg}]$ & NA patients & MA patients & Value of $p$ \\
\hline SBP day-time & $120.23 \pm 14.20$ & $120.25 \pm 7.61$ & 1.00 \\
\hline DBP day-time & $72.13 \pm 7.08$ & $70.12 \pm 7.34$ & 0.46 \\
\hline MAP day-time & $88.17 \pm 7.64$ & $76.83 \pm 6.38$ & 0.43 \\
\hline SBP night-time & $106.77 \pm 7.93$ & $113.62 \pm 10.98$ & $0.03^{*}$ \\
\hline DBP night-time & $60.23 \pm 6.23$ & $62.00 \pm 9.87$ & 0.49 \\
\hline MAP night-time & $75.74 \pm 6.04$ & $79.21 \pm 9.28$ & 0.32 \\
\hline SBP 24 h & $115.25 \pm 8.56$ & $117.87 \pm 8.36$ & 0.42 \\
\hline DBP 24 h & $68.08 \pm 5.89$ & $67.62 \pm 7.91$ & 0.85 \\
\hline MAP 24 h & $83.80 \pm 6.12$ & $84.37 \pm 7.02$ & 0.84 \\
\hline
\end{tabular}

$S B P$ - systolic $B P, D B P$ - diastolic $B P, M A P$ - mean arterial pressure, ${ }^{*} p<0.05$

Table III. Results of univariate logistic regression analysis for microalbuminuria

\begin{tabular}{|lcc|}
\hline Variable & Value of $p$ & Odds ratio $(95 \% \mathrm{Cl})$ \\
\hline Gender & 0.29 & $2.27(0.49-10.53)$ \\
\hline Age [years] & 0.16 & $1.27(0.91-1.77)$ \\
\hline $\begin{array}{l}\text { Pubertal stages: } \\
\text { Tanner I-III vs IV-V }\end{array}$ & 0.16 & $3.19(0.62-16.37)$ \\
\hline $\mathrm{HbA}_{1 \mathrm{c}}(>7.5 \%)$ & $0.04^{*}$ & $1.52(0.97-2.40)$ \\
\hline \begin{tabular}{l}
$\mathrm{BMI}\left[\mathrm{kg} / \mathrm{m}^{2}\right]$ \\
\hline $\begin{array}{l}\text { Systolic BP night-time } \\
\text { [mmHg] }\end{array}$
\end{tabular} & $0.05^{*}$ & $1.26(1.00-1.60)$ \\
\hline $\begin{array}{l}\text { Diastolic BP night-time } \\
\text { [mmHg] }\end{array}$ & 0.49 & $1.10(1.00-1.20)$ \\
\hline $\begin{array}{l}\text { Insulin dose/weight } \\
{[\mathrm{U} / \mathrm{kg}]}\end{array}$ & 0.80 & $1.43(0.09-22.11)$ \\
\hline
\end{tabular}

${ }^{*} p<0.05$

perfusion in early stages of DM1 [14]. It has been confirmed that there is a linear correlation between the onset, or progression of microalbuminuria and 
$\mathrm{HbA}_{1 c}$ values [15]. This finding indicates that there is no definite level of metabolic control $\mathrm{Hb}$ below which metabolic complications are excluded, or inversely, above which exponential rise can occur in the development and the progression of microvascular complications; this means that any change of $\mathrm{HbA}_{1 c}$, with respect to normal values, carries a risk for the development of microalbuminuria (and other microvascular complications) [15].

Obese children, depending on BMI increase, are at a much higher risk of developing hyperlipidaemia and hypertension related to $\mathrm{BMI}$ increase. The risk is proportional. It should be pointed out that obese children without diabetes more frequently have microalbuminuria compared to children with normal weight, which indicates that the presence of early renal glomerular and tubular dysfunction is the consequence of obesity. From this aspect obesity, i.e. increased BMI, carries a higher risk of similar disorders developing in children with DM1 [16]. Our patients with normoalbuminuria had a BMI of $20.85 \mathrm{~kg} / \mathrm{m}^{2}$, whereas the patients with microalbuminuria had a statistically significantly higher BMI $\left(23.59 \mathrm{~kg} / \mathrm{m}^{2}, p<0.05\right)$.

Using ABPM, a non-invasive method, it was possible to detect the earliest BP changes in adolescents with DM1. In our group, the mean value of night-time systolic BP was statistically significantly higher in the patients with microalbuminuria than in those with normoalbuminuria $(p<0.05)$, whereas a statistically significant difference was not found in other mean blood pressure values. Regarding systolic blood pressure during sleep, we obtained similar results to that of Spanish authors who reported a significant correlation between the night-time systolic BP increase and the development and progression of microalbuminuria, also indicating that each $5 \mathrm{mmHg}$ increase of systolic blood pressure resulted in 1.4 times higher increase of urine albumin excretion [17]. Blood pressure increase, especially night-time systolic blood pressure, is associated with intrarenal haemodynamic changes that can cause incipient and clinically evident diabetic nephropathy [17]. Accordingly, we found a statistically highly significant relation between the nighttime blood pressure and albuminuria in our group of patients. Elevations of night-time BP even within the normal range appear to increase the risk for development of diabetic nephropathy.

Urine albumin excretion progresses with diabetes, and likewise a prolonged duration of diabetes leads to the progression of increase in blood pressure [18]. Based on our results, it is evident that the duration of diabetes is statistically significantly associated with night-time systolic and diastolic blood pressure $(p<0.05)$. Most authors consider that, although within the normal value range, the progressive increase of blood pressure can be detected prior to the development of persistent microalbuminuria [19]. Our study also shows that higher insulin doses were associated with higher levels of night-time systolic and diastolic blood pressure. Insulin may induce sodium retention, which may lead to an increase in blood pressure [18]. Future studies will need to consider whether insulin therapy underlies blood pressure changes. In addition, consideration should be given to whether short-term changes in glycaemic control or insulin dose lead to corresponding changes in night-time blood pressure.

The absence of a normal nocturnal dip is a sign of the first noticeable cardiovascular disorder. In healthy individuals a blood pressure decline of at least $10 \%$ occurs during night-time [20]; otherwise, the person could be exposed to a higher risk of target organ damage [20]. In a three-year prospective study Poulsen et al. showed that DM1 children with microalbuminuria developing prior to the onset of incipient nephropathy had a milder BP decline in comparison with diabetic children without microalbuminuria [21]. It is still unclear whether the absence of nocturnal non-dipping precedes increased blood pressure during sleep, or whether its development occurs concomitantly with blood pressure increase; however, nocturnal non-dipping is doubtlessly associated with changes in renal morphology and it is an early predictor of diabetic nephropathy [21]. In our study of children with DM1, in as many as $41.7 \%$ the nocturnal dip was absent, which could be a potential marker of an emerging disorder involving the presence of new complications in a significant number of our patients. In children with microalbuminuria the problem was even more frequent, being present in $62.5 \%$ of cases, whereas in children with normoalbuminuria it was present in $38.5 \%$ of cases. When comparing the normoalbuminuric non-dipping children with the microalbuminuric non-dippers, the following statistically significant differences could be detected: age and BMI were significantly different in children with microalbuminuria (16.9 years vs. 14.6 years; $24.62 \mathrm{~kg} / \mathrm{m}^{2}$ vs. $20.49 \mathrm{~kg} / \mathrm{m}^{2}$ ). The non-dipping children with microalbuminuria had poor metabolic control in comparison with the non-dipping children with normoalbuminuria (9.59\% vs. $8.79 \%$ ), but the difference was not statistically significant.

The prevention of diabetic nephropathy is aimed at early recognition of numerous risk factors that could affect the development of persistent microalbuminuria. In the present study, we have shown that microalbuminuric patients differed from the normoalbuminuric subjects in having higher $\mathrm{HbA}_{1 \mathrm{c}}$, higher BMI and higher systolic night-time blood pressure. Also, the absence of a drop in nocturnal blood pressure has been emphasized in microalbuminuric patients. Our data suggest that the pre- 
vention of diabetic nephropathy should be direct ed toward prevention of obesity and improvement of metabolic control, applying a complex approach when treating adolescent diabetics; thus, it is recommended that $\mathrm{HbA}_{1 \mathrm{c}}$ in childhood should be below $7.5 \%$. In addition, elevations in nocturnal blood pressure could be an important initiating factor of diabetic nephropathy. Ambulatory 24-h measurement can improve the detection of a mild increase in blood pressure concurrent with the development of early diabetic nephropathy.

\section{References}

1. Rossing P. The changing epidemiology of diabetic microangiopathy in type 1 diabetes. Diabetologia 2005; 48: 1439-44

2. Donaghue C, Chiarelli F, Trotta D, Allgrove J, Dahl-Jorgensen K. Microvascular and macrovascular complications. Pediatr Diabetes 2007; 8: 166-7.

3. Mogensen CE. Microalbuminuria, blood pressure and diabetic renal disease: origin and development of ideas. Diabetologia 1999; 42: 263-85.

4. Casani A, Bangstad HJ, Chiarelli F. Detection and management of diabetic glomerulopathy in children and adolescents with insulin-dependent diabetes mellitus: need for improved knowledge and better care. Pediatr Endocrinol Metab 2000; 13: 467-74.

5. Danne T, Kordonouri O, Hovener G, Weber B. Diabetic angiopathy in children. Diab Med 1998; 14: 1012-25.

6. Sochett E, Daneman D. Early diabetes-related complications in children and adolescents with type 1 diabetes. Endocrinol Metab Clin N Am 1999; 28: 865-82.

7. Mauer M, Drummond K. The early natural history of nephropathy in type 1 diabetes. Study design and baseline characteristics of the study participants. Diabetes 2002; 51: 1572-9.

8. Holl RW, Grabert M, Thon A, Heinze E. Urinary excretion of albumin in adolescents with type 1 diabetes. Diabetes Care 1999; 22: 1555-60.

9. Gale EAM. Type 1 diabetes in the young: the harvest of sorrow goes on. Diabetologia 2005; 48: 1435-8.

10. Nordwall M, Bojestig M, Arnqvist HJ, Ludvigsson J. Declining incidence of severe retinopathy and persisting decrease of nephropathy in an unselected population with type 1 diabetes - the Linköping Diabetes Complications Study. Diabetologia 2004; 47: 1266-72.

11. Feldt-Rasmussen B. Microalbuminuria. Int Diabet Monitor 2007; 19: 1-2.

12. Donaghue KC, Fairchild JM, Craig ME, et al. Do all prepubertal years of diabetes duration contribute equally to diabetes complications? Diabetes Care 2003; 26: 1224-9.

13. Gallego PH, Bulsara MK, Frazer F, Lafferty AR, Davis EA Jones TW. Prevalence and risk factors for microalbumin uria in a population - based sample of children and adolescents with T1DM in Western Australia. Pediatr Diabetes 2006; 7: 165-72.

14. Gallego PH, Wiltshire E, Donaghue KC. Identifying children at particular risk of long-term complications. Pediatr Diabetes 2007; 8: 40-8.

15. Mogensen CE, Poulsen PL. Microalbuminuria, glycemic control and blood pressure predicting outcome in diabetes type 1 and type 2. Kidney Int 2004; 66: S40-1.
16. Jorgensen-Dahl K, Larsen JR, Hanssen KF. Atherosclerosis in childhood and adolescent type 1 diabetes: early disease, early treatment? Diabetologia 2005; 48: 1445-53.

17. Lopes CAF, Lerario AC, Mion D Jr, Koch V, Wajchenberg BL, Rosenbloom AL. Ambulatory blood pressure (ABPM) in normontensive adolescents with type 1 diabetes. Pediatr Diabetes 2002; 3: 31-6.

18. Torbjörnsdotter TB, Jaremko GA, Berg UB. Ambulatory blood pressure and heart rate in relation to kidney structure and metabolic control in adolescents with type 1 diabetes. Diabetologia 2001; 44: 865-73.

19. Schultz CJ, Andrew H, Neil W, Neil Dalton R, Konopelska Bahu T, Dunger DB. Blood pressure does not rise before the onset of microalbuminuria in children followed from diagnosis of type 1 diabetes. Diabetes Care 2001; 24: 555-9.

20. Torbjörnsdotter TB, Jaremko GA, Berg UB. Nondipping and its relation to glomerulopathy and hyperfiltration in adolescents with type 1 diabetes. Diabetes Care 2004; 8: 121-3.

21. Poulsen PL, Hansen KW, Mogensen CE. Ambulatory blood pressure in the transition from normo- to microalbuminuria: a longitudinal study in IDDM patients. Diabetes 1994; 43: $1248-53$ 